\title{
Use of Physical Concepts in Musical Instruments and their Use in Cinema
}

\author{
Sanjay Sitaram Patil \\ Department of Physics, K. M. Agrawal College of Arts, Commerce \& Science, Kalyan (W) \\ †Email: sanjay.patil1031969@gmail.com
}

\begin{abstract}
Cinema without music is now a days beyond imagination. Whatever musical instruments used in the making of Cinema are based on Physical Concepts. One of the concepts is frequency. Frequency is nothing but the number of vibrations per second. Different frequencies forms melodious compositions. There are many musical instruments like Pan flute, Sitar, Violin, Harmonium etc are all Physical Concepts dependent musical instruments. How Physical Concepts play important role in the making of such musical instruments is presented in this paper. Unwanted continuous disturbance due to vibrations in air is noise and composition disturbance of different frequencies called sound. Music is sound with a discrete structure. Noise is sound with a continuous structure. Music is composed of sounds with a fundamental frequency and overtones. Noise is composed of sounds with frequencies that range continuously in value from as low as you can hear to as high as you can hear - not necessarily at equal intensity
\end{abstract}

Key words: Physics, Musical instruments, Cinema

\section{Introduction}

Sound waves are first collected in our outer ear (called the auricle or pinna). Then they travel down our ear canal and strike the eardrum, causing it to vibrate. These vibrations are in turn transmitted to our inner ear by the bones of our middle ear (hammer, anvil, and stirrup). Transformation of the vibrations into electrical impulses finally perceived by the brain as sound. A sound wave is the pattern of disturbance caused by the movement of energy traveling through a medium (such as air, water, or any other liquid or solid matter) as it propagates away from the source of the sound. The source is some object that causes a vibration, such as a ringing telephone, or a person's vocal chords. Human ear having capacity to hear a sound of range from $20 \mathrm{~Hz}$ to $20000 \mathrm{~Hz}$ The maximum range of human hearing includes sound frequencies from about 15 to about 18,000 waves, or cycles, per second i.e. $15 \mathrm{~Hz}$ to $18000 \mathrm{~Hz}$. Role of musical instruments is to create various frequencies each frequency having capacity to create disturbance in human ear according to that human brain feels good or bad sound. Sound waves are also 
called as pressure waves. Sound is one kind of longitudinal wave, in which the particles oscillate to and fro in the same direction of wave propagation. Sound is produced when something vibrates. The vibrating body causes the medium (water, air, etc.) around it to vibrate. Vibrations in air are called traveling longitudinalwaves, which we can hear. Sound waves consist of areas of high and low pressure called compressions and rarefactions, respectively.

Question may be in mind that what is music and noise?

Music is ordered sound. While Noise is disordered sound. Music and noise are both mixtures of sound waves of different frequencies. The component frequencies of music are discrete (separable) and rational (their ratios form simple fractions) with a discernible dominant frequency. The component frequencies of noise are continuous (every frequency will be present over some range) and random (described by a probability distribution) with no discernible dominant frequency. Sound is a longitudinal wave, which means the particles of the medium vibrate parallel to the direction of propagation of the wave. A sound wave coming out of a musical instrument, loudspeaker, or someone's mouth pushes the air forward and backward as the sound propagates outward. Musical instruments vibrate naturally at several related frequencies called harmonics. [1]

Musical Instruments Physical Concepts:

1) Harmonium : Physical Concepts and Musical Instruments : There are total of twelve swar of seven are called Shuddh swar they are Sa, Re, Ga, Ma, Pa, Dha, Ni while four are komal are Re, $\mathrm{Ga}$, Dha, Ni and rest one is tivra ma swar

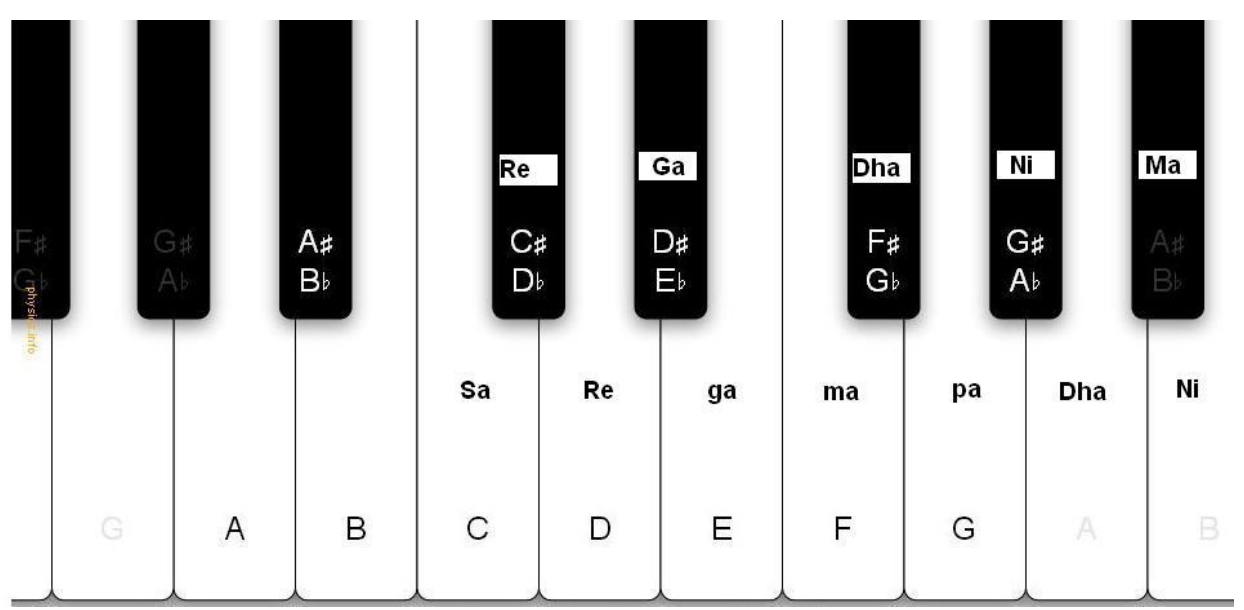

Fig.1: Harmonium Key board 
If one of the frequency is called 'Sa' having frequency $256 \mathrm{~Hz}$ then next frequency will be according to formula (frequency) $\mathrm{x}\left(2^{\frac{1}{12}}\right)$ is $(256) \times\left(2^{\frac{1}{12}}\right)$ which is komal ' $R e^{\text {' }}$

Table 1: Frequency and Swar [2] \& [3]

\begin{tabular}{|c|c|c|}
\hline Swar & Formula & Frequency $(\mathrm{Hz})$ \\
\hline Sa(Shuddh) & $(256) \times\left(2^{\frac{0}{12}}\right)$ & 256 \\
\hline Re(Shuddh) & $(256) \times\left(2^{\frac{1}{12}}\right)$ & 271.22 \\
\hline $\operatorname{Re}($ Komal) & $(256) \times\left(2^{\frac{2}{12}}\right)$ & 287.35 \\
\hline $\mathrm{Ga}$ (Komal) & $(256) \times\left(2^{\frac{3}{12}}\right)$ & 304.43 \\
\hline Ga(Shuddh) & $(256) \times\left(2^{\frac{4}{12}}\right)$ & 322.53 \\
\hline $\mathrm{Ma}$ (Shuddh) & $(256) \times\left(2^{\frac{5}{12}}\right)$ & 341.71 \\
\hline Dha(Komal) & $(256) \times\left(2^{\frac{6}{12}}\right)$ & 362.03 \\
\hline $\mathrm{Pa}$ (Shuddh) & $(256) \times\left(2^{\frac{7}{12}}\right)$ & 383.56 \\
\hline Ni(Shuddh) & $(256) \times\left(2^{\frac{8}{12}}\right)$ & 406.35 \\
\hline Dha(Shuddh) & $(256) \times\left(2^{\frac{9}{12}}\right)$ & 430.53 \\
\hline $\mathrm{Ma}($ Komal) & $(256) \times\left(2^{\frac{10}{12}}\right)$ & 456.14 \\
\hline $\mathrm{Ni}$ & $(256) \times\left(2^{\frac{11}{12}}\right)$ & 483.26 \\
\hline Sa(Shuddh) & $(256) \times\left(2^{\frac{12}{12}}\right)$ & 512 \\
\hline
\end{tabular}

2) Pan Flute: Length (L) of pipe that will be used for making of Pan flute is given as $\mathrm{L}=\frac{\mathrm{v}}{4 f}$; $\mathrm{v}=345 \mathrm{~m} / \mathrm{s}$ for all the lengths

Table 2: Lengths of Pipes and frequencies

\begin{tabular}{|c|c|c|}
\hline Swar & Length of Pipes $(\mathrm{cm})$ & Frequency $(\mathrm{Hz})$ \\
\hline Sa(Shuddh) & 0.336914 & 256 \\
\hline $\operatorname{Re}$ (Shuddh) & 0.318008 & 271.22 \\
\hline Re(Komal) & 0.300157 & 287.35 \\
\hline Ga(Komal) & 0.283316 & 304.43 \\
\hline
\end{tabular}




\begin{tabular}{|c|c|c|}
\hline Ga(Shuddh) & 0.267417 & 322.53 \\
\hline $\mathrm{Ma}($ Shuddh $)$ & 0.252407 & 341.71 \\
\hline $\mathrm{Dha}($ Komal $)$ & 0.23824 & 362.03 \\
\hline $\mathrm{Pa}($ Shuddh $)$ & 0.224867 & 383.56 \\
\hline $\mathrm{Ni}($ Shuddh $)$ & 0.212255 & 406.35 \\
\hline $\mathrm{Dha}$ (Shuddh) & 0.200334 & 430.53 \\
\hline $\mathrm{Ma}($ Komal $)$ & 0.189087 & 456.14 \\
\hline $\mathrm{Ni}$ & 0.178475 & 483.26 \\
\hline $\mathrm{Sa}($ Shuddh $)$ & 0.168457 & 512 \\
\hline
\end{tabular}

It is observed that as frequency increases gradually length of Pipe used for making Pan Flute decreases.

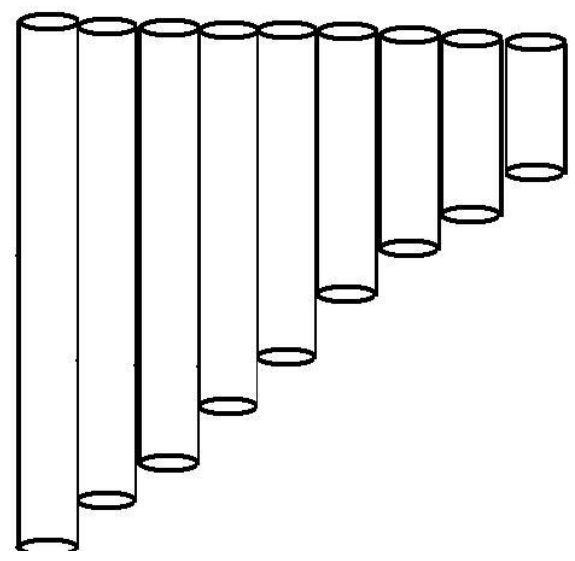

Fig.2: Pan Flute

3) Sitar or Violin: Similary length of wires in Sitar or Violin frequencies can be changed with human fingers and by that melodious combinations of sound as music can be created.

\section{Conclusion}

It is concluded from all the frequencies and their melodious sound concepts of Physics plays key role in the making of Musical instruments. It is also shown from table2 and Fig2 that how it is possible to make Pan flute with formulae given in Physics and according to that lengths of Pipes used in pan flute can be changed by that frequencies can be changed

\section{Reference:}

- https://physics.info/music/

- Physics Reference Book, S. Chand publication

- $\quad$ https://indianmusicart.com/2018/10/28/what-is-shuddh-komal-and-tibra-swar 